\title{
Analysis on the Per Capita Disposable Status of Rural Residents in China and Its Countermeasures
}

\author{
Yuhua Su ${ }^{1,}$, Wei Jiang ${ }^{2, b}$ \\ ${ }^{1}$ School of Mathematics and Computer Science, Hezhou University, Hezhou, Guangxi, 542899, China \\ ${ }^{2}$ Department of Personnel, Hezhou University, Hezhou, Guangxi, 542899, China \\ a107913494@qq.com, b122484412@qq.com
}

Keywords: Time Series Analysis, Disposable Income, Rural Residents

\begin{abstract}
In China's rural social economic statistics, the per capita disposable income of rural residents is a very important statistical indicator. The so-called per capita disposable income of rural residents refers to the average disposable income of rural residents according to the rural population, reflecting the average income of rural residents in a country or region. Among them, "the per capita disposable income of rural residents" refers to the total income of rural residents from various sources in the same year, and the total income after deducting the expenses incurred in income. It is of great significance to analyze the per capita disposable income of rural residents and improve the quality of per capita disposable income of rural residents.
\end{abstract}

\section{Introduction}

In 2015, the per capita disposable income of Chinese farmers reached 11,722 yuan, an average increase of 9.6\% during the "Twelfth Five-Year Plan" period. The per capita disposable income index of rural residents reflects and tests rural social and economic development and the living conditions of farmers. It is the main basis for formulating rural economic policies and monitoring and management of farmers' burdens and formulating measures to increase farmers' income and reduce burdens. Due to the inconsistent survey methods in different regions, the inconsistent indicators and the non-standard accounting standards, the reliability of data sources for farmers' disposable income indicators has been weakened. The data reported and published in various regions are not horizontally comparable, nor can they be evaluated according to uniform standards. Accounting, therefore, it is of great significance to establish and improve the per capita disposable income accounting of rural residents and effectively improve the per capita disposable income data of rural residents.

\section{Problems in the Statistical Investigation and Accounting of Per Capita Disposable Income of Rural Residents in China}

Per capita disposable income of rural residents in China the per capita disposable income of rural residents includes cash income, physical income, food, edible oil, vegetables, meat and poultry products produced by farmers for their own use. After the reform and opening up, the income structure of rural residents has undergone major changes. The nature of income has changed from collective economy to family management. Most farmers are unwilling to expose themselves to the rich, and the income is hidden. The statistical survey and accounting of per capita disposable income of rural residents is becoming more and more difficult.

The gap between the rich and the poor in China's peasants has widened. After the implementation of family management in China, some of the peasants' active business activities have shifted from agriculture to other industries, from working for people to self-employment, and some have become millions, tens of millions or even hundreds of millions of assets. If their income is included, the per capita disposable income indicator for rural residents will be on several floors. Coexisting with this is that there are also some farmers who lack labor, lack of technology, lack of 
funds, and lack of access. They are difficult to manage traditional agriculture, let alone engage in other industries. Their income is very low, growth is slow, and even negative growth. In particular, those who suffer from chronic diseases or serious illnesses will be in trouble. Even if they participate in the new rural cooperative medical care, they will have to bear tens of thousands of yuan in debt a year. According to statistics, the poverty-stricken farmers account for $11.5 \%$ of the total farmers. This disparity between the rich and the poor is still further increasing. Therefore, it is difficult to accurately reflect the actual per capita income of farmers on average.

Administrative intervention, imperfect management system in the performance appraisal of grassroots cadres, the per capita disposable income index of rural residents is generally regarded as a key assessment. For the sake of safeguarding political achievements, some places use the leadership to measure the per capita disposable income of rural residents. Even the number of the beginning of the year, how much is reported at the end of the year; how many people have, how much I report; how much is the leader, how much I will report. The average number is higher than the township average, the average township is higher than the county (city) average, the county (city) average is higher than the provincial average, and the provincial average is higher than the national average. As a result, it is only necessary to organize leadership from the top to the bottom, and to push down the average of the virtual high level. The rural level is the rural grassroots political organization and the rural autonomous organization. Unlike the general enterprises and institutions, its accounting organization has always been responsible for the management of the rural work departments and management departments of the party and the government. Supervision and management are in fact in a state of vacancy, which is also an important reason why the per capita disposable income of rural residents is difficult to get rid of administrative intervention.

\section{Countermeasures for Establishing and Improving the Per Capita Disposable Income Accounting of Rural Residents}

Clarifying work responsibilities, grasping the scope of statistics and accounting methods the per capita disposable income of rural residents is the main statistical indicator that the National Bureau of Statistics requires periodic assessment and review, and there are specific data quality control measures. In order to avoid counting multiple items and ensuring the quality of farmers' income statistics, the people's governments at all levels should assign the functions of statistical survey and accounting of per capita disposable income of rural residents to local statistical departments, and publish and use them by cities, counties (districts). The per capita disposable income data of rural residents shall be directly investigated by the statistical departments at all levels according to the national statistical system, and the data after examination and approval shall prevail. At the township level, according to the plan of the National Bureau of Statistics, the survey on the per capita disposable income of rural residents in each township was carried out, and a survey system for townships was established. In view of the objective situation of the disparity between the rich and the poor in rural areas, the classification and calculation of the per capita disposable income of rural residents is divided into special rich households, special poor households and ordinary households. Ordinary households are the mainstream, which can best reflect the actual affluence of most farmers. The special rich households and special poor households also have their special significance. It is especially important for people to understand the rural status quo and formulate and adjust the rural and rural policies. The scope of statistics can only be actual rural households in rural areas. Farmers who have left their homes should not be included in the statistics and accounting fields, and should be accounted for by their new settlements.

In terms of performance appraisal, the role of rural grassroots organizations and grassroots cadres in the evaluation of peasants' income is mainly based on guiding the work of unified service organizations, such as variety improvement and agricultural technology promotion, and farmland water conservancy. Infrastructure construction, prevention and control of pests and diseases, advancement of rural industrialization, construction of agricultural professional cooperative organizations, and organization and implementation of disaster relief and disaster relief, etc., have done a good job of contributing to the growth of farmers' income. Otherwise, even if farmers' 
incomes increase, also has nothing to do with them. Therefore, the assessment of the achievements of grassroots cadres can only be achieved by assessing the actual performance of the unified management services, and can truly assess the actual achievements and contributions of the cadres' work, and then draw the attention of cadres at all levels to do practical things, engage in services, and benefit the people. In order to make the statistical investigation and accounting of per capita disposable income of rural residents get rid of administrative intervention and control.

The sample method is used for accounting, the grading burden statistical survey, and the accounting statistics system to carry out the 100-sample survey method, one for each township, one point. The advantage of this approach is that it is more scientific and adapts to the requirements of the new situation, but the problem is insufficient manpower and insufficient funds. The statistical system is responsible for business counseling, and establishes 100 household survey points by village or by film. Grassroots statistics and financial personnel are responsible for organizing systematic records, statistics and accounting, and stressing authenticity, comparability, systemicity, accuracy and scientificity to ensure rural areas. The per capita disposable income accounting data is credible and reliable. All levels of finance must allocate a certain amount of special funds for the development of this level of investigation program, statistical survey and level account book printing, data network transmission, data super summary, equipment purchase and maintenance, as well as business training, work start and so on. Governments at all levels should, in accordance with the principle of financial grading, include special funds in the annual budget, and properly address the requirements for the statistical departments of all levels to carry out the accounting for the per capita disposable income of rural residents.

Strengthening legal supervision, investigating and accounting according to law, and counting the people's governments at all levels and the statistical departments at all levels, upholding the idea of seeking truth from facts, firmly establishing the concept of statistical accounting according to law, strictly implementing the "Statistics Law" and "Accounting Law", and clarifying statistics and finance. The legal supervision responsibility of the department, clarify the regulations on rural statistics, accounting and financial supervision, and through the improvement of laws and regulations, the rural grassroots statistical investigation and accounting are gradually incorporated into the track of the legal system, and the legal supervision of the authenticity of rural statistical accounting is implemented. Effectively ensure the legality and accuracy of statistical surveys and accounting data sources.

We should continue to increase fiscal and people's livelihood spending, and further strengthen its income-increasing effect on urban and rural residents. Since the fiscal and livelihood expenditure has a significant crowding effect on the service consumption of urban and rural residents, the strengthening of the people's biochemical orientation of fiscal expenditure is a realistic choice to promote the upgrading of residents' consumption structure. Considering that the crowding-in effect of fiscal and livelihood expenditure on residents' service consumption is mainly achieved through the disposable income of residents, in the process of promoting the construction of modern financial system, we must focus on strengthening the income-increasing effect of fiscal and people's livelihood expenditure on residents. To this end, we should continue to increase investment in key areas of people's livelihood such as education, health care, social security and employment, focus on improving the allocation and use efficiency of people's livelihood, and improve public education, health care, social security, social employment, etc. The supply level of services, on the one hand, enhances the ability of people to participate in the initial distribution of national income by increasing the level of human capital, and on the other hand, by increasing the transfer payment, the degree of benefit of residents from the redistribution of national income is increased, and efforts are made to improve residents, especially The disposable income level of rural residents continues to improve the pattern of national income distribution and continuously improve the spending power of urban and rural residents.

\section{Effective Measures to Increase the Disposable Income of Rural Residents}

First, promote full employment and increase farmers' income from non-agriculturalization. It is 
necessary to take advantage of the opportunity of Jiangsu's industrialization and urbanization to accelerate the development of rural labor to entrepreneurship and employment in urban and secondary and tertiary industries, directly driving the optimization of farmers' income structure. According to the actual situation of rural economic development in the three major regions of southern Jiangsu, northern Jiangsu and central Jiangsu, in the process of economic development transformation and upgrading, we should pay more attention to relying on the actual resources of each region, scientifically plan the development layout of rural secondary and tertiary industries, and realize resource complementarity and industrial linkage. Farmers can achieve non-agricultural employment to provide a better environment and create more employment opportunities; increase labor skills training for rural surplus labor, help them expand employment areas, improve employability; gradually break the dual household registration system, and strive to create a fair urban-rural integration Employment policy and environment; provide employment guidance to existing rural non-agricultural workers, enhance awareness of safeguarding their own labor rights; further implement various policies and measures to encourage the development of small and medium-sized enterprises, optimize the development environment of small and medium-sized enterprises, and cultivate a number of characteristics of small and medium-sized enterprises Industrial clusters allow farmers to participate more in the process of regional industrialization and urbanization, and more directly realize the diversification and non-agriculturalization of income sources from the whole value chain and the entire industrial chain.

Second, innovate the rural management system and tap the potential of farmers to increase their income. First of all, on the basis of accelerating the reform of the transfer mechanism of rural land management rights, we should develop appropriate scale operations of various forms such as land concentration, cooperative operation, and unified service according to local conditions, and develop new rural collective economy and farmer cooperative economic organizations to accelerate Agricultural industrialization management, increase the added value of agricultural and sideline products, and expand the channels for farmers to increase their income. Secondly, we should speed up the transformation and upgrading of agriculture, cultivate more new types of agricultural management entities, encourage migrant workers to transfer land to new agricultural management entities, promote advanced agricultural practical technologies, develop agricultural socialization services, and upgrade new agricultural management entities to operate agriculture. Through the development of multi-functional agriculture, farmers will be guided to participate in rural tourism, agricultural service industry, agricultural product processing, rural e-commerce and other fields to foster new growth points for farmers' family business income. Finally, in view of the fact that the rural residents in Jiangsu Province have a relatively low proportion of net property income, accelerate the reform of the rural collective property rights system, so that farmers can obtain income from collective assets, and make the value-added income of collective assets become the important income of rural residents. Source: Breaking the urban-rural dual-real estate system and policy restrictions, on the basis of rationally establishing the complete property rights of farmers' real estate, realizing the capitalization, marketization and commercialization of farmers' real estate, so that more farmers can sell, rent, mortgage, and inherit in housing. Increase the property income in the circulation. Implementing the same treatment of urban and rural construction land use and urban collective land participation in urban development in the process of urbanization, so that farmers can obtain more land value-added income and raise farmers' property income level (Yang Guofan, 2015).

Third, improve the social security system and increase the net income of farmers. Increase fiscal transfer payments, comprehensively implement various agricultural subsidy policies, gradually increase direct subsidy standards for agriculture and farmers; improve agricultural subsidies, promote the agricultural subsidy policy that combines the GSP with precision subsidies, and increase direct subsidies for agriculture Targeted, improve the efficiency of pure farmers engaged in agricultural production and management (Zhu et al., 2014). Promote the integration of urban and rural social security systems, effectively improve the level of rural social security, and achieve the equalization of public services for urban and rural residents; establish a good rural financial support 
system and commercial insurance system, and play a positive role in rural financial and commercial insurance to increase farmers' income; A unified minimum living security system and a low-income farmer assistance system to increase farmers' transfer income.

\section{References}

[1] Zhu Hanqing. A Comparative Study of Income Structure of Farmers with Different Income Levels__Based on Statistical Data of Zhejiang Province from 1995 to 2012[J]. Development Research, 2014(12): 86-91.

[2] Yang Canming, Guo Huifang, Sun Qunli. An Empirical Analysis of the Sources of Farmers' Income in China__ On the Countermeasures to Increase Farmers' Income[J]. Finance \& Trade in Economics, 2007(2): 24-26.

[3] Yuan Liang, Tang Hongtao, Chen Gongjun. Correspondence Analysis and Application of T-TEST in China's Residents' Income Structure-Based on the Empirical Study of China Statistical Yearbook 2006[J]. Technology Economics and Management Research, 2007(5) ): 51-54.

[4] Zeng Lingqiu. Analysis of Farmers' Income Structure and Farmers' Income Increase[J]. Productivity Research, 2007(3): 21-22. Du Huazhang: Analysis of the Differences in Income Structure of Rural Residents in Jiangsu Province

[5] Ye Caixia, Shi Guoqing, Chen Shaojun. An Empirical Analysis of the Influence of Regional Differences on Farmers' Income Structure[J]. Economic Issues, 2010(10): 103-107. 